\title{
CRNDE Contributes Cervical Cancer Progression by Regulating miR-4262/ZEBI Axis
}

This article was published in the following Dove Press journal: OncoTargets and Therapy

\section{Lu Ren \\ Shaoqin Yang \\ Qinxue Cao \\ Jun Tian}

Department of Obstetrics and Gynecology, Huaihe Hospital of Henan University, Kaifeng, 47500I Henan,

People's Republic of China
Correspondence: Jun Tian Department of Obstetrics and Gynecology, Huaihe Hospital of Henan University, Kaifeng 47500I, Henan,

People's Republic of China

Tel +86 37I-23906579

Emaildfwmex@163.com
Background: Cervical cancer is a lethal gynecologic cancer in women. Long non-coding RNA colorectal neoplasia differentially expressed (LncRNA CRNDE) was recognized as a significant oncogene in multiple cancers. However, the functional role of CRNDE in cervical cancer remains poorly explored.

Methods: The expression of CRNDE, microRNA-4262 (miR-4262) and zinc-finger E-box binding homeobox 1 (ZEB1) in cervical cancer tumors and cells was detected by quantitative real-time polymerase chain reaction (qRT-PCR). Colony formation and 3-(4,5-dimethyl2-thiazolyl)-2,5-diphenyl-2-H-tetrazolium bromide (MTT) were performed to detect cell viability. Flow cytometry and caspase- 3 activity assay were conducted to evaluate cell apoptosis. The interaction between miR-4262 and CRNDE or ZEB1 was verified by dualluciferase reporter system. Transwell assay was employed to evaluate cell migration and invasion. The relative protein expression was assessed by Western blot.

Results: CRNDE and ZEB1 were up-regulated, while miR-4262 was down-regulated in cervical cancer tissues and cells. We found that CRNDE sponged miR-4262 and ZEB1 was a target of miR-4262. In addition, miR-4262 inhibitor abolished CRNDE silencing-induced repression on cell proliferation, EMT, migration, invasion and promotion on cell apoptosis. Furthermore, ZEB1 rescued the effects of miR-4262 overexpression or CRNDE deletion on cervical cancer progression. Our data showed that CRNDE targeted miR-4262 to regulate ZEB1 expression in cervical cancer cells. Besides, CRNDE expedited cervical cancer progression through wnt/ $\beta$-catenin pathway via sponging miR-4262 and altering ZEB1 expression.

Conclusion: Our findings demonstrated that CRNDE facilitated the progression of cervical cancer through activation of wnt/ $\beta$-catenin pathway by regulating miR-4262/ZEB1 axis, representing a prospective targeted therapy for cervical cancer.

Keywords: CRNDE, miR-4262, ZEB1, wnt/ $\beta$-catenin pathway, cervical cancer

\section{Introduction}

Cervical cancer is a common gynaecological malignancy with high incidence, mortality and low survival rate. ${ }^{1,2}$ Although the diagnosis and treatment of cervical cancer have achieved improvements, the 5-year survival rate of metastatic cervical cancer is still low. ${ }^{3,4}$ Therefore, it is of significance to explore the mechanism of cervical cancer progression. Long non-coding RNA colorectal neoplasia differentially expressed (CRNDE) was involved in the development of a variety of cancers. $^{5,6}$ For example, CRNDE functioned as oncogene in hepatocellular carcinoma to facilitate cell growth, invasion and epithelial to mesenchymal transition (EMT) by interacting with miR-217 to regulate MAPK1 expression. ${ }^{7}$ CRNDE acted 
as a sponge of miR-641 to expedite cell growth and block cell apoptosis by improving CDK6 expression in nonsmall cell lung cancer. ${ }^{8}$ Moreover, CRNDE was proved to function as an oncogene in the progression of cervical cancer. ${ }^{9}$ Therefore, it is imperative to disclose the biological mechanism of CRNDE in cervical cancer progression.

MicroRNAs (miRNAs) play significant roles in various diseases by participating in cell behavior regulation through binding to the messenger RNA (mRNA) and altering gene expression. ${ }^{10-12}$ Dysregulation of miRNAs was observed in the pathogenesis of multiple diseases. For instance, microRNA-4262 (miR-4262) was involved in chondrocyte growth, autophagy and apoptosis by interacting with SIRT1 through the activation of $\mathrm{PI} 3 \mathrm{~K} / \mathrm{AKT} / \mathrm{mTOR}$ pathway. ${ }^{13}$ However, the function of miR-4262 in cancers was controversial. Up-regulation of miR-4262 could accelerate the progression of hepatocellular carcinoma. ${ }^{14}$ Oppositely, miR-4262 acted as tumor suppressor to attenuate cell proliferation and induce cell apoptosis by regulating GALNT4 in colon cancer. ${ }^{15}$ A previous study indicated that miR-4262 participated in the progression of cervical cancer. ${ }^{16}$ However, the pathological mechanism of miR-4262 in cervical cancer requires further exploration.

The transcription factor zinc finger E-box-binding homeobox 1 (ZEB1), as a key EMT activator, can promote cancer progression. For instance, ZEB1 was involved in the progression of breast cancer. ${ }^{17}$ The wnt $/ \beta$-catenin signaling pathway, composed of a series of oncogene and antioncogene proteins, plays a vital role in the process of EMT. ${ }^{18}$ A previous research reported that PAK6 activated the wnt/ $\beta$-catenin signaling pathway to promote the development of cervical cancer. ${ }^{19}$ Moreover, ZEB1 served as an oncogene in cervical cancer. ${ }^{20}$ Thus, we explored the functional role and underlying mechanism of ZEB1 in cervical cancer progression.

In this study, we aimed to clarify the molecular mechanism of CRNDE in cervical cancer cell growth, invasion, EMT and apoptosis. Moreover, the levels of CRNDE, miR-4262 and ZEB1 in cervical cancer were measured. Besides, rescue experiments were performed to reveal the regulatory mechanism of CRNDE, miR4262 and ZEB1 in cervical cancer progression.

\section{Materials and Methods}

\section{Patient Samples}

Forty cervical cancer tumor tissues and the corresponding normal tissues were collected from 40 cervical cancer patients by surgery at Huaihe Hospital of Henan University. All patients signed the written informed consents. Our protocols were approved by Ethics Committee of Huaihe Hospital of Henan University (No. KF2019226).

\section{Cell Transfection}

C-33A, Hela, SiHa, CasKi cells and normal cervical epithelial cells ECT1/E6E7 were purchased from ATCC (Manassas, VA, USA) and cultured in DMEM medium (Gibco, Carlsbad, CA, USA) supplemented with $10 \%$ FBS (Gibco). Small hairpin RNA (shRNA) targeting CRNDE (sh-CRNDE) and the negative control (sh-NC), pcDNA, CRNDE and ZEB1 overexpression vectors were synthesized by Genepharma (Shanghai, China). MiR-4262 mimic (MiR-4262) and the control (miR-NC), miR-4262 inhibitor (anti-miR-4262) and the negative control inhibitor (anti-miR-NC) were purchased from RIBOBIO (Guangzhou, China). Cell transfection was performed in Hela and SiHa cells using Lipofectamine 2000 (Invitrogen, Carlsbad, CA, USA).

\section{Quantitative Real-Time Polymerase Chain Reaction (qRT-PCR)}

Total RNA was extracted from cervical cancer tumors, normal tissues and the cells using TRIzol reagent (Invitrogen). The cDNA for CRNDE, miR-4262 and ZEB1 was synthesized by All-in-One ${ }^{\mathrm{TM}}$ cDNA Synthesis Kit (FulenGen, Guangzhou, China). Subsequently, SYBR green (Applied Biosystems, Foster City, CA, USA) was used for qRT-PCR. Glyceraldehyde-3-phosphate dehydrogenase (GAPDH) and U6 were employed as internal references. The primers for CRNDE, miR-4262, ZEB1, GAPDH and U6 were listed as follows: CRNDE, (Forward, 5'-TGTCAAGGGTTTGAAC TGCTAAT-3'; Reverse, 5'- GATAAGCATGCTACAAGC TCTGACA-3'); miR-4262 (Forward, 5'-AAAGATTGCT GGGTCACACC-3'; Reverse, 5'-TGTCAAAAGGGAAAT GAGGC-3'); ZEB1 (Forward, 5'-GATGATGAATGCGAGT CAGATGC-3'; Reverse, 5'-ACAGCAGTGTCTTGTTGT TGT-3'); GAPDH, (Forward, 5'-AGGTCGGTGTGAACG GATTTG-3'; Reverse, 5'-GGGGTCGTTGATGGCAACA -3'); U6, (Forward, 5'-ACCCTGAGAAATACCCTCACAT -3'; Reverse, 5'-GACGACTGAGCCCCTGATG-3'). The relative expression level was estimated by $2^{-\Delta \Delta C t}$ method.

\section{Dual-Luciferase Reporter Assay}

Wild type CRNDE (CRNDE-WT), ZEB1 (ZEB1 3'UTRWT), mutant type CRNDE (CRNDE-MUT), ZEB1 
(ZEB1 3'UTR-MUT) luciferase vectors were constructed. Next, the vectors were co-transfected with miR-4262 or miR-NC into Hela and $\mathrm{SiHa}$ cells. Finally, the luciferase activities were measured using a luminometer.

\section{Colony Formation Assay}

Hela and SiHa cells mixed with top agar were placed on the base agar in 6-well plates. After incubation for 2 weeks, the cells were stained with $0.1 \%$ crystal violet (Sigma, St. Louis, MO, USA). Finally, the colonies $\geq 0.5 \mathrm{~mm}$ were counted by a microscope.

\section{3-(4,5-Dimethyl-2-Thiazolyl)- 2,5-Diphenyl-2-H-Tetrazolium Bromide (MTT) Assay}

Hela and SiHa cells were plated on the 96-well plates. After incubation for 24, 48 and $72 \mathrm{~h}, 10 \mu \mathrm{L}$ MTT (Beyotime, Shanghai, China) was added to each well and incubated for another $4 \mathrm{~h}$. Then, the cells were cultured with $100 \mu \mathrm{L}$ dimethyl sulfoxide (DMSO; Sigma) for 2 h. Finally, the optical density (OD) value at $490 \mathrm{~nm}$ was detected by a spectrophotometer.

\section{Flow Cytometry}

Hela and SiHa cells were plated on the 24-well plates and cultured for $48 \mathrm{~h}$. Then, the cells were re-suspended, collected and stained using fluorescein isothiocyanate tagged Annexin V (Annexin V-FITC)/propidium iodide (PI) detection kit (Invitrogen). The apoptotic rate was counted by a flow cytometer.

\section{Detection of Caspase-3 Activity}

Caspase-3 activity was evaluated by caspase-3 activity assay kit (Beyotime). In brief, transfected Hela and SiHa cells were seeded on the 24-well plate and incubated for $48 \mathrm{~h}$. Then, the cells were collected and caspase- 3 activity assay kit was employed to determine caspase-3 activity.

\section{Western Blot Assay}

Western blot was conducted as previously reported. ${ }^{21}$ The primary antibodies against ZEB1, Ki-67, PCNA, Bcl-2, Bax, MMP9, N-cadherin, E-cadherin, Vimentin, Snail, $\beta$ catenin, GSK-3 $\beta$, c-myc, cyclin D1 and GAPDH were purchased from Abcam (Cambridge, MA, USA) and HRP- conjugated secondary antibody was obtained from Sangon (Shanghai, China).

\section{Transwell Assay}

For transwell migration assay, Hela and SiHa cells were placed on the upper chamber non-coated with Matrigel. For transwell invasion assay, the upper chamber was treated with Matrigel. After incubation for $48 \mathrm{~h}$, the migrated and invaded cells at lower chamber were stained with $0.1 \%$ crystal violet and counted by a microscope.

\section{Statistical Analysis}

Data from at least three repeated experiments were presented as means \pm standard deviation (SD). Statistical analysis was carried out using SPSS software and GraphPad Prism 7. The correlation between miR-4262 and CRNDE or ZEB1 was analyzed by Pearson's correlation coefficient. $P$ value less than $0.05(P<0.05)$ was considered statistically significant.

\section{Results}

\section{CRNDE Was Increased, While miR-4262 Was Decreased in Cervical Cancer Tissues and Cells}

We investigated the role of CRNDE and miR-4262 in cervical cancer by qRT-PCR and further validated the interaction between them using dual-luciferase reporter assay. CRNDE was upregulated in cervical cancer tissues and cells (C-33A, Hela, SiHa, CasKi) compared with normal tissues and cells (ECT1/E6E7) (Figure 1A and B). Besides, downregulation of CRNDE arrested cell cycle at G1 period (Supplement Figure 1) and inhibited cell proliferation (Supplement Figure 2), implying the oncogenic role of CRNDE in cervical cancer. What is more, CRNDE expression of cervical cancer patients was associated with clinical features (Table 1). As expected, CRNDE expression was dramatically increased by CRNDE overexpression and decreased by CRNDE knockdown in Hela and SiHa cells (Figure 1C). StarBase predicted that CRNDE harbored the binding sites with miR-4262 (Figure 1D). Reduced luciferase activity in Hela and SiHa cells cotransfected with CRNDE-WT and miR-4262 confirmed the prediction (Figure $1 \mathrm{E}$ and $\mathrm{F}$ ). Interestingly, the expression of miR-4262 was down- 

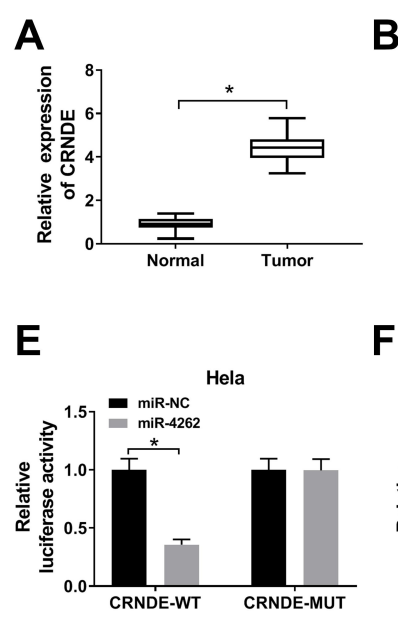

F
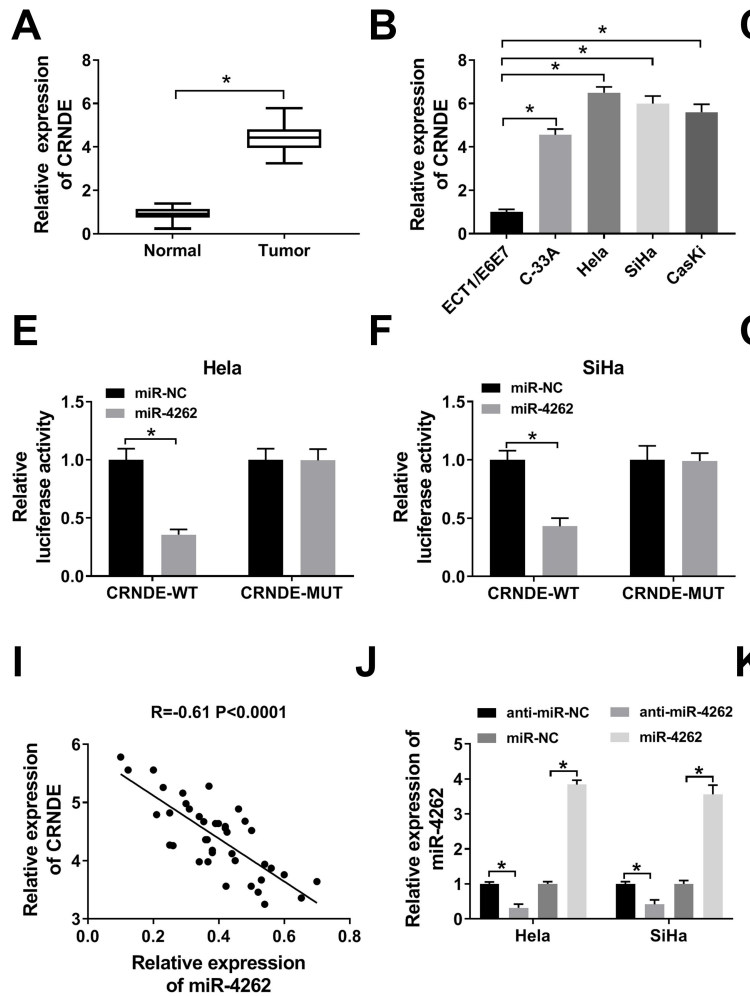

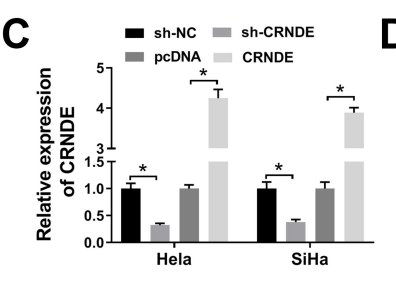

G

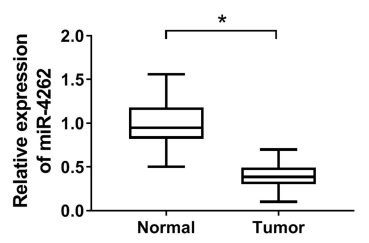

K
D

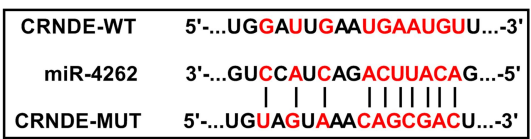

H

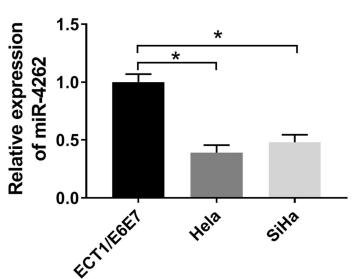

Figure I CRNDE directly interacted with miR-4262. (A and B) CRNDE expression was detected by qRT-PCR in cervical cancer tumors and cells (C-33A, Hela, $\mathrm{SiHa}, \mathrm{CasKi}$ ) as well as the normal tissues and cells (ECTI/E6E7). (C) CRNDE expression was detected using qRT-PCR in Hela and SiHa cells transfected with shNC, pcDNA, sh-CRNDE and CRNDE. (D) Prediction of the putative binding sites between CRNDE and miR-4262 using StarBase. (E and F) Luciferase activity of Hela and SiHa cells co-transfected with CRNDE-WT or CRNDE-MUT and miR-4262 or miR-NC was determined by dual-luciferase reporter assay. (G and $\mathbf{H}$ ) The expression of miR-4262 in cervical cancer tumors and cells as well as the normal counterparts was detected by qRT-PCR. (I) Analysis of the correlation between CRNDE and miR-4262 by Person's correlation coefficient $(R=-0.61, P<0.0001)$. (J) Detection of miR-4262 expression in Hela and SiHa cells transfected with antimiR-NC, miR-NC, anti-miR-4262 and miR-4262 by qRT-PCR. (K) Measurement of miR-4262 expression in Hela and SiHa cells transfected with sh-NC, pcDNA, shCRNDE and CRNDE by qRT-PCR. $* P<0.05$.

regulated in cervical cancer tissues and cells (C-33A, Hela, SiHa, CasKi) compared with normal tissues and cells (ECT1/E6E7), suggesting the suppressive role of miR-4262 in cervical cancer (Figure $1 \mathrm{G}$ and $\mathrm{H}$ ). MiR4262 expression of cervical cancer patients was related to FIGO stage (Table 2). We also discovered that CRNDE was inversely correlated with miR-4262 ( $\mathrm{R}=$ $-0.61, P<0.0001$ ) (Figure 1I). Moreover, miR-4262 expression was enhanced in Hela and SiHa cells transfected with miR-4262, and reduced in cells transfected with miR-4262 inhibitor (Figure 1J). As exhibited in Figure $1 \mathrm{~K}$, abundance of CRNDE inhibited miR-4262 expression, whereas deficiency of CRNDE promoted the expression of miR-4262. These data indicated that CRNDE negatively regulated miR-4262 expression in cervical cancer.

\section{MiR-4262 Inhibitor Neutralized CRNDE Silencing-Induced Repression on Cell Proliferation and Promotion on Cell Apoptosis in Cervical Cancer}

The regulatory effects of CRNDE/miR-4262 axis on cervical cancer growth were explored. The expression of miR-4262 was elevated by CRNDE silencing, which was declined by miR-4262 inhibitor (Figure 2A). The number of colonies was down-regulated by CRNDE silencing, which was reversed by miR-4262 inhibitor (Figure 2B). In addition, miR-4262 inhibitor attenuated the suppressive effects of CRNDE silencing on the proliferation of cervical cancer cells (Figure $2 \mathrm{C}$ and D). Meanwhile, knockdown of CRNDE inhibited the expression of cell proliferation-related proteins including KI-67 and PCNA, which were abolished by 
Table I Association Between Clinical Features and CRNDE Expression of Cervical Cancer Patients $(n=40)$

\begin{tabular}{|c|c|c|c|c|}
\hline \multirow[t]{2}{*}{ Parameters } & \multirow[t]{2}{*}{ Case } & \multicolumn{2}{|c|}{ CRNDE Expression $^{a}$} & \multirow[t]{2}{*}{$P$ value } \\
\hline & & High $(n=20)$ & Low $(n=20)$ & \\
\hline \multicolumn{5}{|l|}{ Age (years) } \\
\hline$\leq 50$ & 22 & 12 & 10 & 0.252 \\
\hline$>50$ & 18 & 8 & 10 & \\
\hline \multicolumn{5}{|l|}{ Tumor size } \\
\hline$\leq 4 \mathrm{~cm}$ & 25 & 9 & 16 & $0.022^{*}$ \\
\hline$>4 \mathrm{~cm}$ & 15 & II & 4 & \\
\hline \multicolumn{5}{|l|}{$\begin{array}{l}\text { Lymph node } \\
\text { metastasis }\end{array}$} \\
\hline No & 22 & 15 & 7 & $0.019 *$ \\
\hline Yes & 18 & 5 & 13 & \\
\hline \multicolumn{5}{|l|}{ FIGO stage } \\
\hline I-Ila & 24 & 8 & 16 & $0.010 *$ \\
\hline IIb-III & 16 & 12 & 4 & \\
\hline
\end{tabular}

Notes: $* P<0.05 ;{ }^{a}$ Using median expression level of CRNDE as cutoff.

Table 2 Association Between Clinical Features and miR-4262 Expression of Cervical Cancer Patients $(n=40)$

\begin{tabular}{|c|c|c|c|c|}
\hline \multirow[t]{2}{*}{ Parameters } & \multirow[t]{2}{*}{ Case } & \multicolumn{2}{|c|}{ miR-4262 Expression ${ }^{a}$} & \multirow[t]{2}{*}{$P$ value } \\
\hline & & High $(n=27)$ & Low $(n=13)$ & \\
\hline \multicolumn{5}{|l|}{ Age (years) } \\
\hline$\leq 50$ & 26 & 17 & 9 & 0.697 \\
\hline$>50$ & 14 & 10 & 4 & \\
\hline \multicolumn{5}{|l|}{ Tumor size } \\
\hline$\leq 4 \mathrm{~cm}$ & 19 & 14 & 5 & 0.427 \\
\hline$>4 \mathrm{~cm}$ & 21 & 13 & 8 & \\
\hline \multicolumn{5}{|l|}{$\begin{array}{l}\text { Lymph node } \\
\text { metastasis }\end{array}$} \\
\hline No & 22 & 17 & 5 & 0.145 \\
\hline Yes & 18 & 10 & 8 & \\
\hline \multicolumn{5}{|l|}{ FIGO stage } \\
\hline I-Ila & 25 & 20 & 5 & $0.029 *$ \\
\hline IIb-III & 15 & 7 & 8 & \\
\hline
\end{tabular}

Notes: $* P<0.05$; ${ }^{2}$ Using median expression level of miR-4262 as cutoff.

miR-4262 inhibitor (Figure 2E and F). Cell apoptosis induced by CRNDE silencing was suppressed by miR4262 inhibitor (Figure 2G). Synchronously, CRNDE knockdown boosted the expression of pro-apoptotic protein Bax and blocked the expression of antiapoptotic protein Bcl-2. However, miR-4262 inhibitor reversed these effects (Figure $2 \mathrm{H}$ and $\mathrm{I}$ ). Besides, the relative caspase- 3 activity was enhanced by CRNDE knockdown, which was reversed by miR-4262 inhibitor (Figure 2J). Taken together, CRNDE regulated cervical cancer cell growth and apoptosis by interacting with miR-4262.

\section{CRNDE Enhanced Cervical Cancer Cell Migration, Invasion and EMT by Sponging miR-4262}

Hela and SiHa cells were transfected with sh-NC, shCRNDE, sh-CRNDE+anti-miR-NC or sh-CRNDE+antimiR-4262 to further explore the mechanism of CRNDE/ miR-4262 axis in cervical cancer development. As illustrated in Figure $3 \mathrm{~A}$ and $\mathrm{B}$, cell migration and invasion were repressed by CRNDE knockdown. However, the repressive effect was inversed by miR-4262 inhibitor. Next, Western blot was exploited to detect the expression of EMT markers. The results revealed that the expression of E-cadherin boosted by CRNDE silencing was inhibited by miR-4262 inhibitor, while the expression of $\mathrm{N}$-cadherin, Vimentin and Snail displayed the opposite trend (Figure 3C and D). All findings demonstrated that CRNDE could regulate cell migration, invasion and EMT by interacting with miR-4262 in cervical cancer.

\section{ZEBI Acted as a Target of miR-4262}

Based on the prediction by StarBase, miR-4262 comprised the potential binding sites of ZEB1 (Figure 4A). Luciferase activity was decreased in Hela and $\mathrm{SiHa}$ cells co-transfected with ZEB1 3'UTR-WT and miR4262, but not in ZEB1 3'UTR-MUT group, validating the interaction between ZEB1 and miR-4262 (Figure $4 \mathrm{~B}$ and $\mathrm{C})$. Moreover, the mRNA and protein expression of ZEB1 were extremely higher in cervical cancer tissues (Figure 4D and E) and cells (C-33A, Hela, $\mathrm{SiHa}, \mathrm{CasKi})$ compared with normal tissues and cells (ECT1/E6E7) (Supplement Figure 3). ZEB1 expression of cervical cancer patients was related to FIGO stage (Table 3). ZEB1 was negatively correlated with miR4262 in cervical cancer tissues $(\mathrm{R}=-0.60, P<0.0001)$ (Figure 4F). ZEB1 protein expression was up-regulated in cervical cancer cells (Figure 4G). Furthermore, the mRNA and protein expression of ZEB1 were promoted by miR-4262 inhibitor and suppressed by miR-4262 mimic (Figure 4H-K). Besides, we also detected the effect of CRNDE overexpression or lower expression on the expression of ZEB1. As shown in 


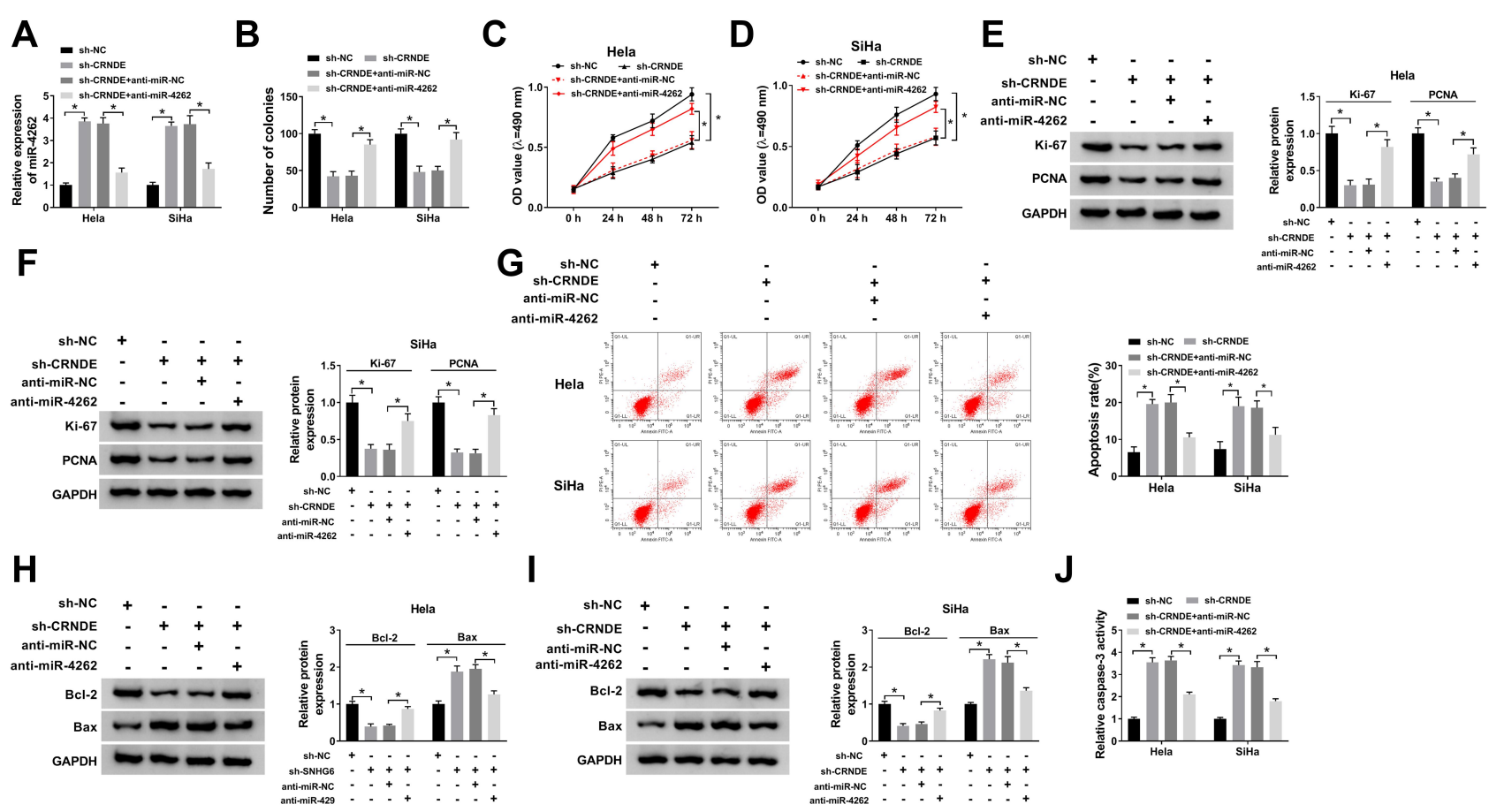

Figure 2 MiR-4262 inhibitor restored CRNDE silencing-induced suppressive effects on cervical cancer progression. Hela and SiHa cells were transfected with sh-NC, shCRNDE, sh-CRNDE+anti-miR-NC and sh-CRNDE+anti-miR-4262. (A) Examination of miR-4262 expression in transfected cells by qRT-PCR. (B) Analysis of colonies of transfected cells by colony formation assay. (C and D) Cell viability was detected by MTT assay. (E and F) The protein expression of Ki-67 and PCNA was detected by Western blot. (G) Cell apoptosis was determined by flow cytometry. (H and I) Detection of apoptosis-associated proteins including Bcl-2 and Bax using Western blot. (J) Identification of caspase-3 activity of transfected cells by caspase-3 activity kit. $* P<0.05$.

A

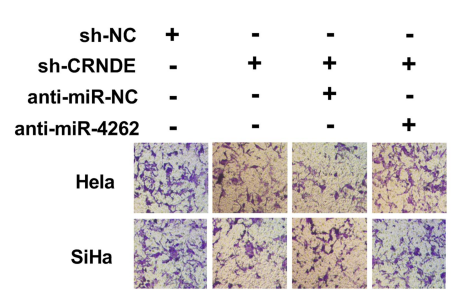

C

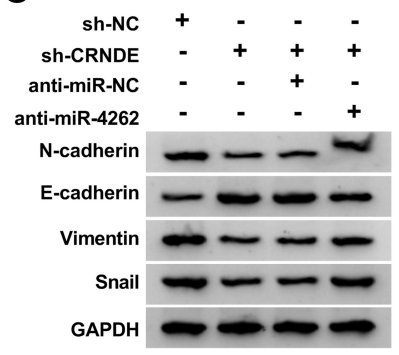

B

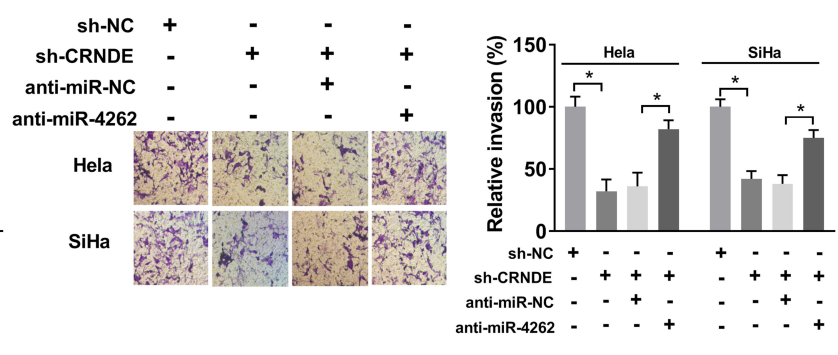

D

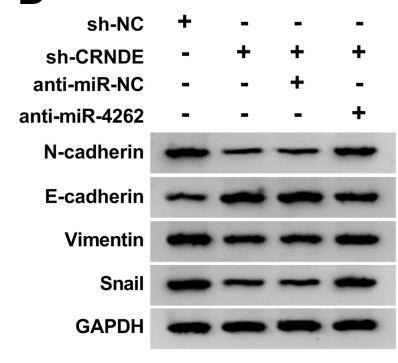

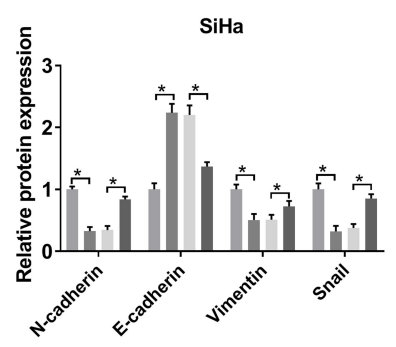

Figure 3 MiR-4262 inhibitor abrogated CRNDE silencing-mediated inhibition on cervical cancer cell migration, invasion and EMT. Hela and SiHa cells were transfected with sh-NC, sh-CRNDE, sh-CRNDE+anti-miR-NC and sh-CRNDE+anti-miR-4262. (A and B) Cell migration and invasion were assessed by transwell assay. (C and D) The protein expression of $\mathrm{N}$-cadherin, E-cadherin, Vimentin and Snail was analyzed by Western blot. $* P<0.05$. 


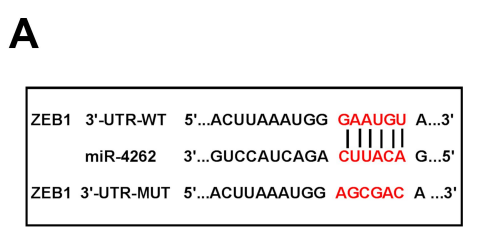

$\mathbf{E}$

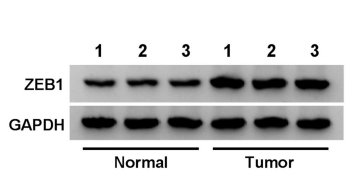

H

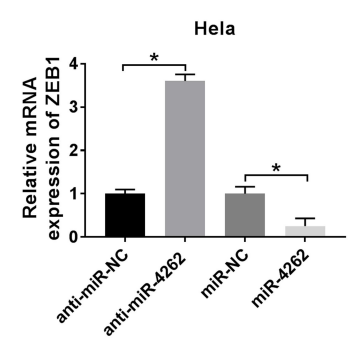

B

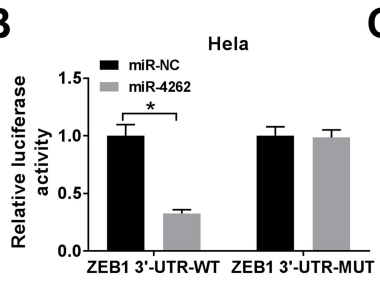

$\mathbf{F}$
C

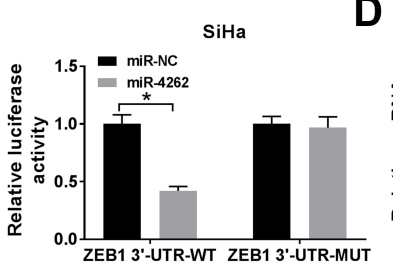

D

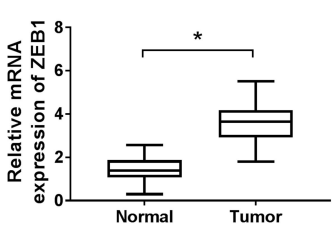

G
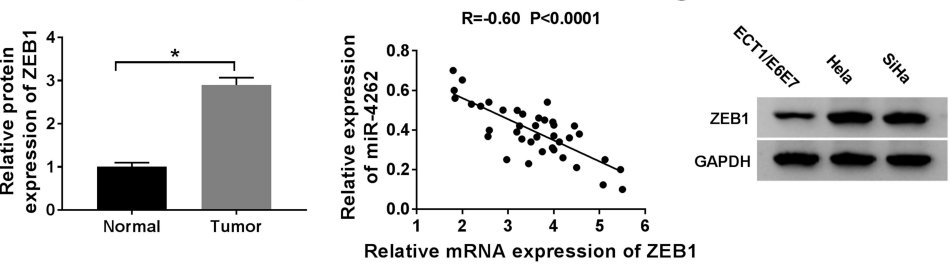

J

Hela

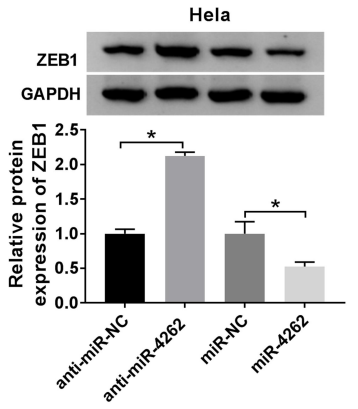

K
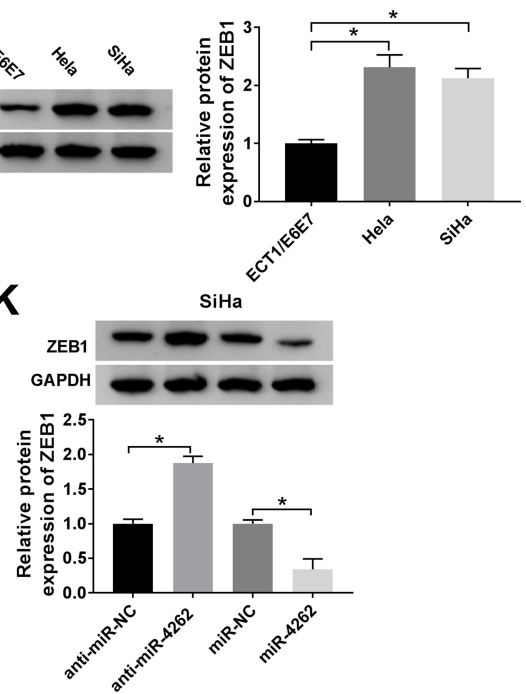

Figure 4 ZEBI was a target of miR-4262. (A) The putative binding sites between ZEBI and miR-4262 were predicted by StarBase. (B and C) Dual-luciferase reporter assay was conducted to evaluate the luciferase activity of Hela and SiHa cells co-transfected with ZEBI 3'UTR-WT or ZEBI 3'UTR-MUT and miR-4262 or miR-NC. (D and E) ZEBI mRNA and protein expression in cervical cancer tumors compared with normal tissues were examined by qRT-PCR and Western blot. (F) Analysis of the correlation between ZEBI and miR-4262 by Person's correlation coefficient $(R=-0.60, P<0.000 I)$. (G) Protein expression of ZEBI in cervical cancer cells and normal cells was measured by Western blot. (H-K) Detection of ZEBI mRNA and protein expression in Hela and SiHa cells transfected with anti-miR-NC, anti-miR-4262, miR-NC and miR-4262 using qRT-PCR and Western blot. $* P<0.05$.

Supplement Figure 4, CRNDE overexpression promoted the expression of ZEB1 while CRNDE knockdown inhibited ZEB1 expression. Taken together, ZEB1 was negatively regulated by miR-4262 in cervical cancer.

\section{MiR-4262 Regulated Cell Proliferation and Apoptosis by Targeting ZEBI in Cervical Cancer}

To explore the underlying mechanism of miR-4262 in cervical cancer, Hela and $\mathrm{SiHa}$ cells were transfected with miR-NC, miR-4262, miR-4262+Vector or miR4262+ZEB1. As exhibited in Figure 5A-C, the mRNA and protein expression of ZEB1 reduced by miR-4262 were reversed by ZEB1. In addition, the number of colonies was decreased by miR-4262, which was blocked by ZEB1 overexpression (Figure 5D). Moreover, restoration of ZEB1 rescued miR-4262mediated inhibition effect on cell viability (Figure $5 \mathrm{E}$ and F) and promotion effect on cell apoptosis (Figure 5I). Meanwhile, the proliferative protein $\mathrm{Ki}-67$ and PCNA expression were suppressed by miR-4262 upregulation, which were reversed by up-regulation of ZEB1 (Figure $5 \mathrm{G}$ and $\mathrm{H}$ ). Moreover, restoration of ZEB1 recovered miR-4262-induced inhibition of Bcl-2 expression and promotion of Bax expression (Figure 5J and $\mathrm{K})$. As expected, the relative caspase-3 activity elevated by miR-4262 was abolished by ZEB1 overexpression (Figure 5L). We concluded that miR-4262 could affect cervical cancer cell proliferation and apoptosis by targeting ZEB1.

\section{MiR-4262 Modulated the Migration, Invasion and EMT of Cervical Cancer Cells by Targeting ZEBI}

Next, transwell assay and Western blot were performed to illuminate the effects of miR-4262/ZEB1 axis on cervical cancer cell migration, invasion and EMT. As 
Table 3 Association Between Clinical Features and ZEBI Expression of Cervical Cancer Patients $(n=40)$

\begin{tabular}{|c|c|c|c|c|}
\hline \multirow[t]{2}{*}{ Parameters } & \multirow[t]{2}{*}{ Case } & \multicolumn{2}{|c|}{ ZEB I Expression ${ }^{\mathbf{a}}$} & \multirow[t]{2}{*}{$P$ value } \\
\hline & & High $(n=17)$ & Low $(n=23)$ & \\
\hline \multicolumn{5}{|l|}{ Age (years) } \\
\hline$\leq 50$ & 20 & 10 & 10 & 0.337 \\
\hline$>50$ & 20 & 7 & 13 & \\
\hline \multicolumn{5}{|l|}{ Tumor size } \\
\hline$\leq 4 \mathrm{~cm}$ & 23 & 12 & II & 0.150 \\
\hline$>4 \mathrm{~cm}$ & 17 & 5 & 12 & \\
\hline \multicolumn{5}{|l|}{$\begin{array}{l}\text { Lymph node } \\
\text { metastasis }\end{array}$} \\
\hline No & 24 & 8 & 16 & 0.151 \\
\hline Yes & 16 & 9 & 7 & \\
\hline \multicolumn{5}{|l|}{ FIGO stage } \\
\hline I-Ila & 22 & 13 & 9 & $0.019 *$ \\
\hline IIb-III & 18 & 4 & 14 & \\
\hline
\end{tabular}

Notes: ${ }^{*} P<0.05 ;{ }^{a} U$ sing median expression level of ZEBI as cutoff.

illustrated in Figure 6A and B, restoration of ZEB1 reversed miR-4262-mediated suppression on cell migration and invasion in Hela and SiHa cells. In addition, miR-4262 boosted the expression of E-cadherin and inhibited the expression of MMP9, Vimentin and $\mathrm{N}$-cadherin. However, ZEB1 reversed these effects of miR-4262 on the expression of EMT markers (Figure $6 \mathrm{C}$ and D). Overall, ZEB1 restored the repressive effects of miR-4262 on cell migration, invasion and EMT in cervical cancer.

\section{ZEBI Overexpression Reversed the Effects of CRNDE Deletion on Cervical Cancer Cells}

To explore whether CRNDE exerted its function through regulating ZEB1, Hela and $\mathrm{SiHa}$ cells were transfected with sh-NC, sh-CRNDE, sh-CRNDE + Vector, or sh-CRNDE + ZEB1, respectively. As shown in Supplement Figure 5A-B, the inhibition effect of CRNDE deletion on cell proliferation was abolished by ZEB1 overexpression. Cell apoptosis was promoted by CRNDE knockdown, which was partially reversed by ZEB1 upregulation in Hela and SiHa cells (Supplement Figure 5C). Furthermore, our data indicated that the suppressive effects of CRNDE knockdown on cell migration and invasion were partially blocked by ZEB1 overexpression in cervical cancer cells (Supplement Figure 5D-E). These findings demonstrated that CRNDE knockdown inhibited cervical cancer progression partially through regulating ZEB1 expression.

\section{CRNDE Targeted miR-4262 to Regulate ZEBI Expression in Cervical Cancer}

In order to figure out the interaction among CRNDE, miR-4262 and ZEB1, Hela and SiHa cells were transfected with miR-NC, miR-4262, pcDNA and CRNDE. The mRNA and protein expression of ZEB1 were inhibited by miR-4262, and the inhibitive effect of miR-4262 was reversed by CRNDE (Figure 7A-D). These results demonstrated that CRNDE could regulate ZEB1 expression by sponging miR-4262 in cervical cancer.

\section{CRNDE Activated the $\mathrm{Wnt} / \beta$-Catenin Pathway by Regulating miR-4262/ZEB I Axis}

The molecular mechanism of CRNDE in cervical cancer cell development was further investigated by transfecting sh-NC, sh-CRNDE, sh-CRNDE+anti-miR-NC, sh-CRNDE+anti-miR-4262, sh-CRNDE+Vector and shCRNDE+ZEB10 into Hela and SiHa cells. As exhibited in Figure 8A and B, depletion of CRNDE blocked the expression of $\beta$-catenin, $c$-myc and cyclin D1 and enhanced the expression of GSK-3 $\beta$. However, these regulatory effects of CRNDE knockdown were reversed by miR-4262 inhibitor or ZEB1 overexpression. Collectively, CRNDE could regulate cell behaviors by interacting with miR-4262/ZEB1 axis through the wnt/ $\beta$ catenin pathway.

\section{Discussion}

Accumulating evidences demonstrated that CRNDE was a critical treatment and prognosis biomarker in different cancers, such as glioma, breast cancer and gastric cancer. ${ }^{22-24}$ For example, CRNDE was overexpressed in hepatocellular carcinoma and abundance of CRNDE contributed to cell proliferation and invasion by sponging miR-337-3p and enhancing SIX1 expression. ${ }^{25}$ Similarly, CRNDE promoted pancreatic cancer cell growth and metastasis by increasing IRS1 level via sponging miR-384. ${ }^{26}$ Enhanced expression of CRNDE accelerated the malignancy of breast cancer by 


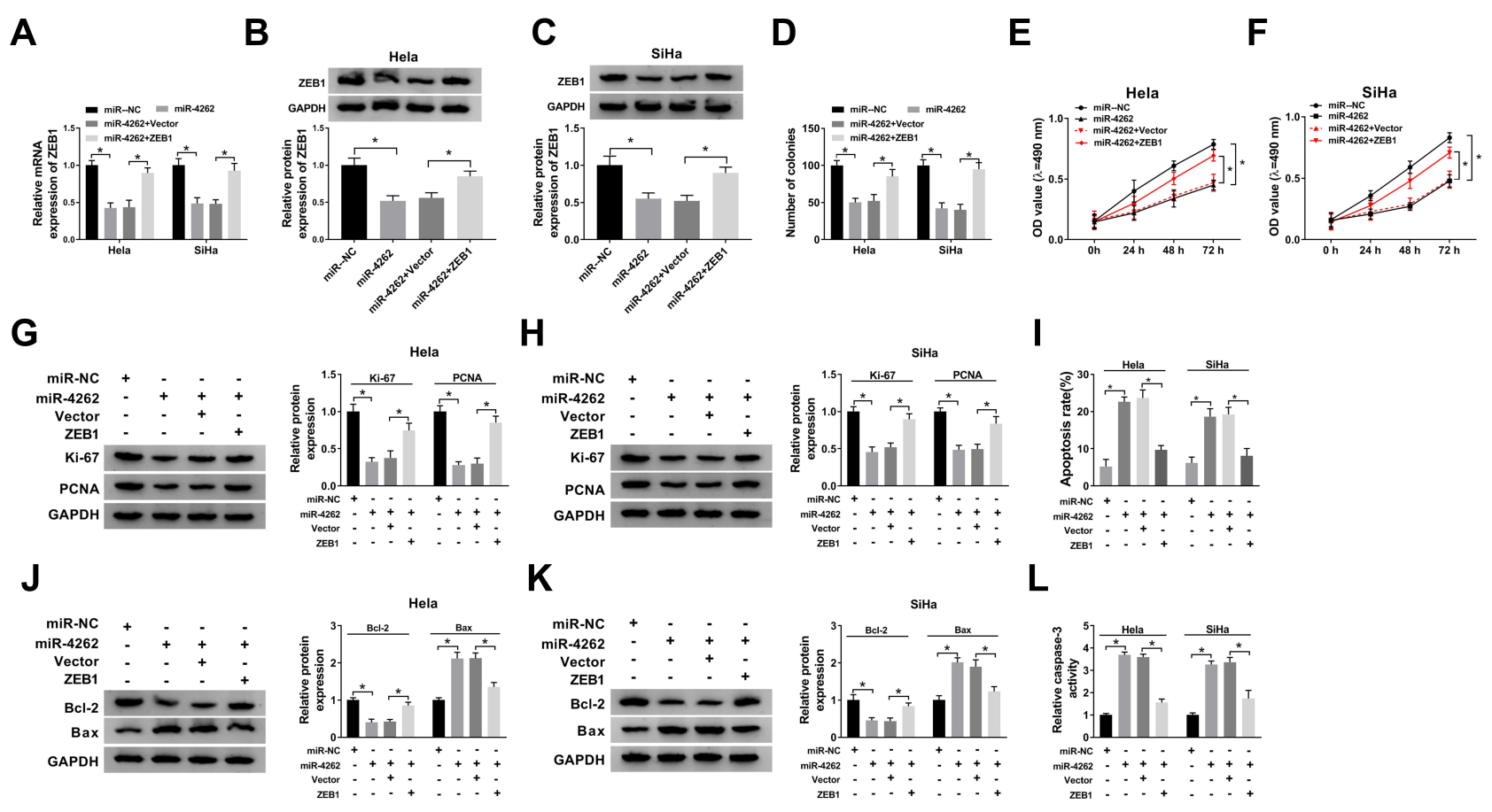

Figure 5 ZEBI attenuated miR-4262-mediated inhibition on cell proliferation and promotion on cell apoptosis in cervical cancer. Hela and SiHa cells were transfected with miR-NC, miR-4262, miR-4262+Vector and miR-4262+ZEBI. (A-C) Detection of ZEBI mRNA and protein expression in transfected cells using qRT-PCR and Western blot. (D) Measurement of colonies of transfected cells by colony formation assay. ( $\mathbf{E}$ and $\mathbf{F}$ ) Cell viability was detected by MTT assay. (G and $\mathbf{H}$ ) Western blot was performed to examine the expression of Ki-67 and PCNA. (I) Cell apoptosis was measured by flow cytometry. (J and $\mathbf{K}$ ) Detection of apoptosis-associated proteins including Bcl-2 and Bax using Western blot. (L) Examination of caspase- 3 activity of transfected cells by caspase- 3 activity kit. $* P<0.05$.

sponging miR-136 through the activation of $\mathrm{Wnt} / \beta$ catenin signaling. ${ }^{27}$ Moreover, elimination of CRNDE alleviated cell survival, invasion, EMT and boosted apoptosis in squamous cell carcinoma. ${ }^{28}$ In this study, we found that CRNDE was upregulated in cervical cancer tissues and cells. Furthermore, CRNDE knockdown suppressed cell proliferation, migration, invasion, EMT, and promoted cell apoptosis in cervical cancer.

Online database StarBase predicted that miR-4262 might be a target of CRNDE. Previous studies indicated that miR-4262 was a significant modulator in multiple diseases, including cancers. ${ }^{16,29,30}$ For instance, overexpression of miR-4262 was reported to facilitate cutaneous melanoma cell growth and invasion by targeting KLF6 and activating EGFR/p21 pathway. ${ }^{31}$ Similarly, miR-4262 contributed to the progression of breast cancer by interacting with KLF6/ KLF15. ${ }^{32}$ Conversely, miR-4262 inhibited the development of gastric cancer and colon cancer by targeting CD163 and GALNT4, respectively. ${ }^{33,34}$ In this research, miR-4262 was decreased in cervical cancer tissues and cells. Moreover, the expression of
miR-4262 was negatively regulated by CRNDE in cervical cancer cells. Besides, miR-4262 inhibitor could reverse the effects of CRNDE knockdown on the progression of cervical cancer.

Next, we found that ZEB1 was a target of miR-4262 and negatively regulated by miR-4262. We hypothesized that CRNDE facilitated cervical cancer development by regulating miR-4262/ZEB1 axis. Our data showed that restoration of ZEB1 inversed miR-4262-mediated suppression effects on the progression of cervical cancer. Besides, CRNDE exerted its function by regulating ZEB1 expression via targeting miR-4262. By measuring the protein expression of $\beta$-catenin, GSK-3 $\beta$, c-myc and cyclin D1, we discovered that CRNDE could activate the wnt/ $\beta$-catenin pathway by regulating miR-4262/ ZEB1 axis.

In summary, CRNDE accelerated cell survival, EMT, migration, invasion, but attenuated cell apoptosis by regulating miR-4262/ZEB1 axis through activation of wnt/ $\beta$ catenin pathway in cervical cancer. Our study elucidated the molecular mechanism for cervical cancer development and provided the potential biomarkers for cervical cancer therapy. 
A

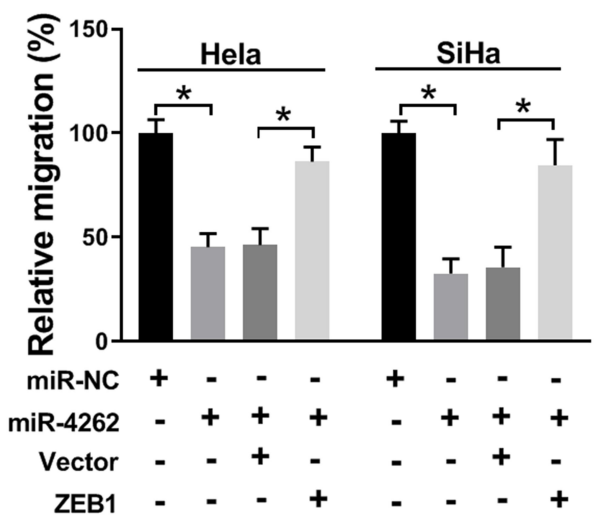

C
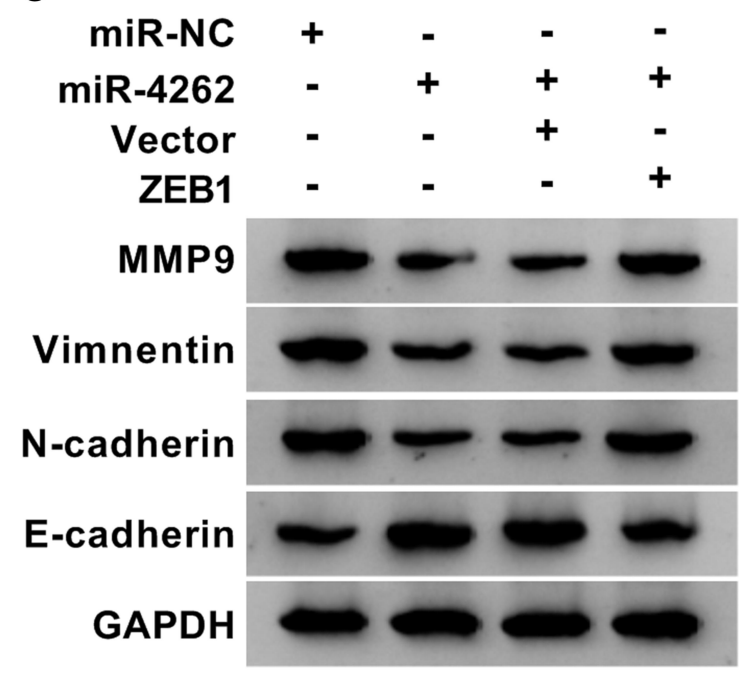

D

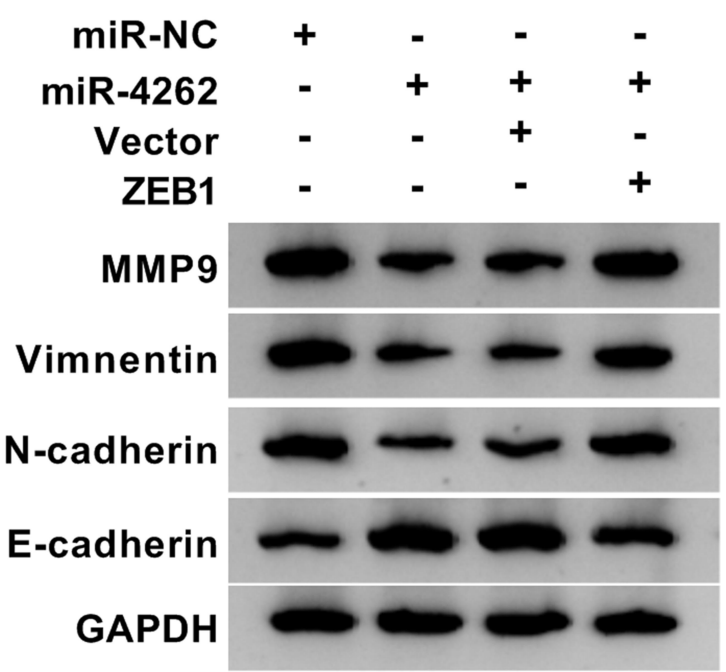

B

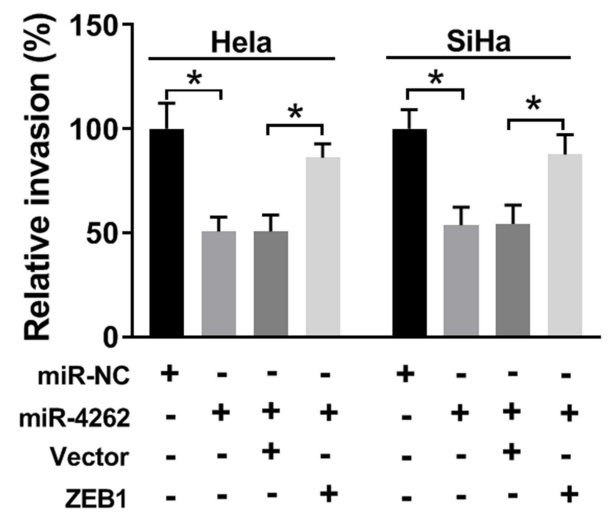

\section{Hela}

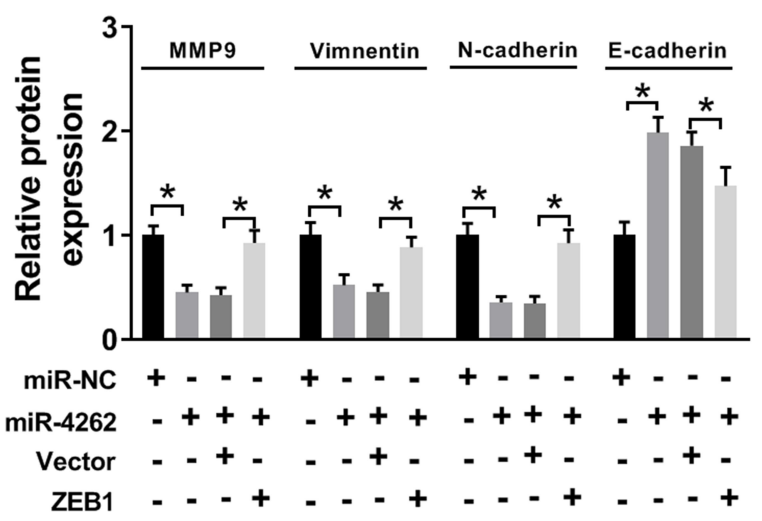

$\mathrm{SiHa}$

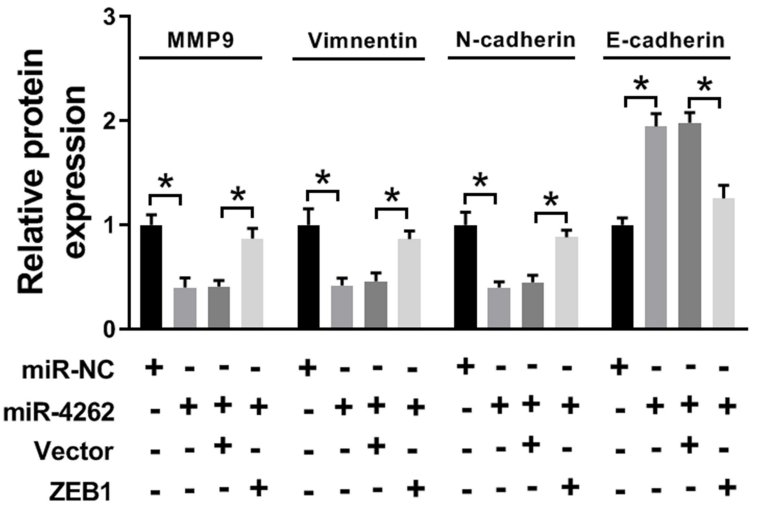

Figure 6 ZEBI abolished miR-4262-induced suppression on the migration, invasion and EMT of cervical cancer cells. Hela and SiHa cells were transfected with miR-NC, miR-4262, miR-4262+Vector and miR-4262+ZEBI. (A and B) Transwell assay was applied to evaluate cell migration and invasion. (C and D) Protein expression of MMP9, Vimentin, $\mathrm{N}$-cadherin and $\mathrm{E}$-cadherin was analyzed by Western blot. $* P<0.05$. 

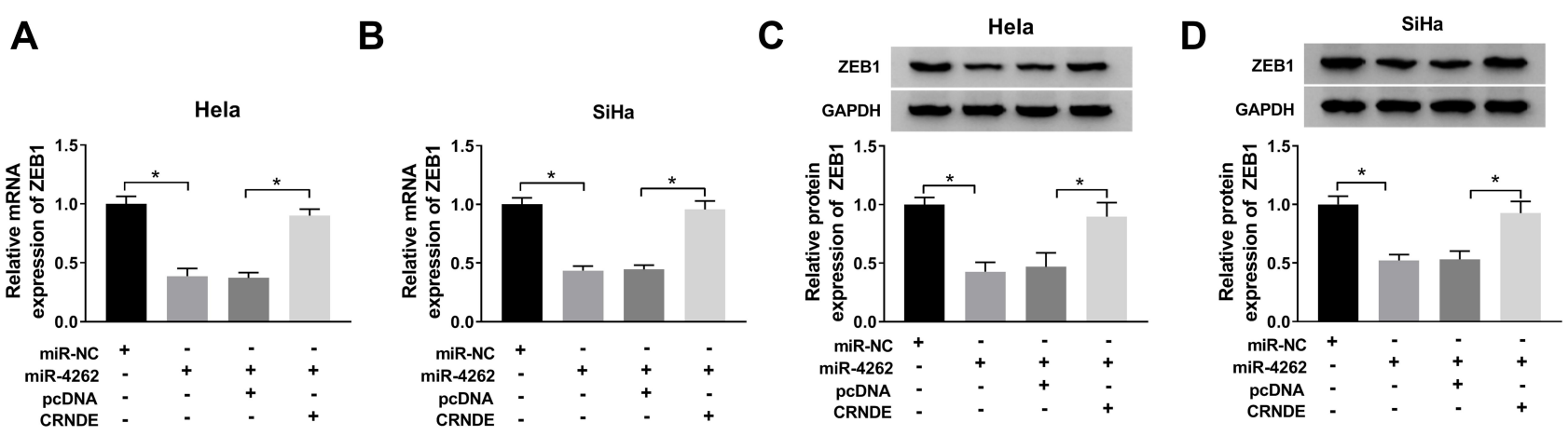

Figure 7 CRNDE regulated ZEBI expression by sponging miR-4262. Hela and SiHa cells were transfected with miR-NC, miR-4262, pcDNA and CRNDE. (A and B) Evaluation of ZEBI mRNA expression in transfected cells using qRT-PCR. (C and D) Analysis of ZEBI protein expression in transfected cells by Western blot. *P<0.05.

A

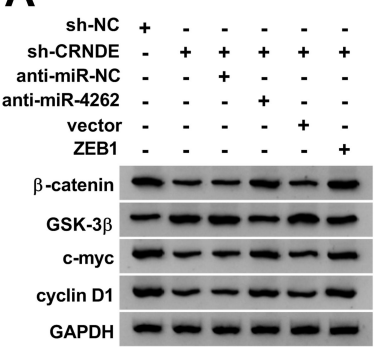

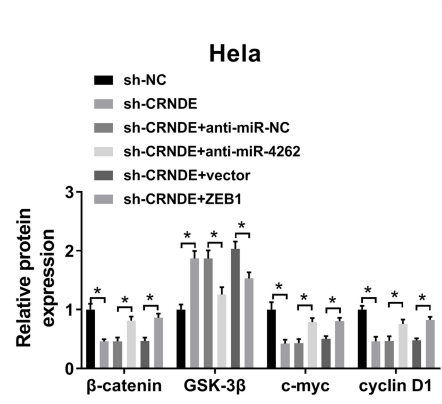

B

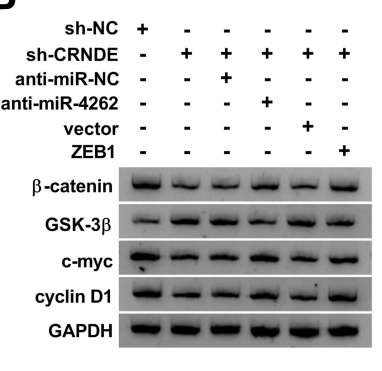

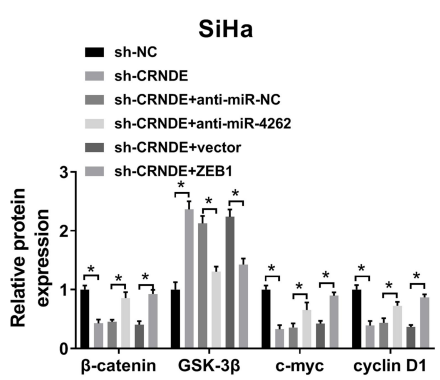

Figure 8 CRNDE activated the wnt/ $\beta$-catenin pathway by targeting miR-4262/ZEBI axis in cervical cancer. Hela and SiHa cells were transfected with sh-NC, sh-CRNDE, shCRNDE+anti-miR-NC, sh-CRNDE+anti-miR-4262, sh-CRNDE+Vector and sh-CRNDE+ZEBI0. (A and B) Detection of the protein expression of $\beta$-catenin, GSK-3 $\beta$, c-myc and cyclin DI in transfected cells by Western blot. $* P<0.05$.

\section{Disclosure}

The authors declare that they have no financial conflicts of interest.

\section{References}

1. Matsuo K, Fullerton ME, Moeini A. Treatment patterns and survival outcomes in patients with cervical cancer complicated by complete uterine prolapse: a systematic review of literature. Int Urogynecol J. 2016;27(1):29-38. doi:10.1007/s00192-015-2731-8

2. Valenti G, Vitale SG, Tropea A, et al. Tumor markers of uterine cervical cancer: a new scenario to guide surgical practice? Updates Surg. 2017;69(4):441-449. doi:10.1007/s13304-017-0491-3

3. Uyar D, Rader J. Genomics of cervical cancer and the role of human papillomavirus pathobiology. Clin Chem. 2014;60(1):144-146. doi:10.1373/clinchem.2013.212985

4. Giavedoni ME, Staringer L, Garrido R, et al. Experience with concurrent chemoradiotherapy treatment in advanced cervical cancer: results from a hospital in Argentina. $E$ Cancer Med Sci. 2019;13:919. doi:10.3332/ecancer.2019.919

5. Zhang JJ, Fan LP. Long non-coding RNA CRNDE enhances cervical cancer progression by suppressing PUMA expression. Biomed Pharmacother. 2019;117:(108726. doi:10.1016/j.biopha.2019.108 726

6. Meng $\mathrm{Y}$, Li Q, Li $\mathrm{L}$, et al. The long non-coding RNA CRNDE promotes cervical cancer cell growth and metastasis. Biol Chem. 2017;399(1):93-100. doi:10.1515/hsz-2017-0199
7. Wang H, Ke J, Guo Q, et al. Long non-coding RNA CRNDE promotes the proliferation, migration and invasion of hepatocellular carcinoma cells through miR-217/MAPK1 axis. J Cell Mol Med. 2018;22(12):5862-5876. doi:10.1111/jcmm.13856

8. Fan Y-F, Yu Z-P, Cui X-Y. IncRNA colorectal neoplasia differentially expressed (CRNDE) promotes proliferation and inhibits apoptosis in non-small cell lung cancer cells by regulating the miR-641/CDK6 axis. Med Sci Monitor. 2019;25:(2745-2755. doi:10.12659/MSM.913420

9. Bai X, Wang W, Zhao P, et al. LncRNA CRNDE acts as an oncogene in cervical cancer through sponging miR-183 to regulate CCNB1 expression. Carcinogenesis. 2020;41(1):111-121. doi:10.1093/carcin/bgz166

10. Liu K, Liu S, Zhang W, et al. miR-494 promotes cell proliferation, migration and invasion, and increased sorafenib resistance in hepatocellular carcinoma by targeting PTEN. Oncol Rep. 2015;34 (2):1003-1010. doi:10.3892/or.2015.4030

11. Sun X, Liu Y, Li M, et al. Involvement of miR-485-5p in hepatocellular carcinoma progression targeting EMMPRIN. Biomed Pharmacother. 2015;72:(58-65. doi:10.1016/j.biopha.2015.04.008

12. Zhang Q, Li Y, Zhao M, et al. MiR-494 acts as a tumor promoter by targeting CASP2 in non-small cell lung cancer. Sci Rep. 2019;9 (1):3008. doi:10.1038/s41598-019-39453-2

13. Sun W, Li Y, Wei S. miR-4262 regulates chondrocyte viability, apoptosis, autophagy by targeting SIRT1 and activating PI3K/AKT/ mTOR signaling pathway in rats with osteoarthritis. Exp Ther Med. 2018;15(1):1119-1128. doi:10.3892/etm.2017.5444

14. Lu S, Wu J, Gao Y, et al. MicroRNA-4262 activates the NF-kappaB and enhances the proliferation of hepatocellular carcinoma cells. Int J Biol Macromol. 2016;86:(43-49. doi:10.1016/j.ijbiomac.20 16.01.019 
15. Weng L, Ma J, Jia Y-P, et al. MiR-4262 promotes cell apoptosis and inhibits proliferation of colon cancer cells: involvement of GALNT4. Am J Transl Res. 2018;10(12):3969-3977.

16. Feng J. Upregulation of MicroRNA-4262 targets kaiso (ZBTB33) to inhibit the proliferation and EMT of cervical cancer cells. Oncol Res. 2018;26(8):1215-1225. doi:10.3727/096504017X15021536183526

17. Jiang X, Zhou Y, Sun AJ, et al. NEAT1 contributes to breast cancer progression through modulating miR-448 and ZEB1. J Cell Physiol. 2018;233(11):8558-8566. doi:10.1002/jcp.26470

18. Liu XH, Sun M, Nie FQ, et al. Lnc RNA HOTAIR functions as a competing endogenous RNA to regulate HER2 expression by sponging miR-331-3p in gastric cancer. Mol Cancer. 2014;13(1):92. doi:10.1186/1476-4598-13-92

19. Yang Q, Zhao Y, Chen Y, et al. PAK6 promotes cervical cancer progression through activation of the Wnt/ $\beta$-catenin signaling pathway. Oncol Lett. 2020;20(3):2387-2395. doi:10.3892/ol.2020.11797

20. Xu J, Wang H, Wang $\mathrm{H}$, et al. The inhibition of miR-126 in cell migration and invasion of cervical cancer through regulating ZEB1. Hereditas. 2019;156:11. doi:10.1186/s41065-019-0087-7

21. Chen R, Xia W, Wang X, et al. Upregulated long non-coding RNA SBF2-AS1 promotes proliferation in esophageal squamous cell carcinoma. Oncol Lett. 2018;15(4):5071-5080. doi:10.3892/ol.2018.7968

22. Ding C, Han F, Xiang H, et al. LncRNA CRNDE is a biomarker for clinical progression and poor prognosis in clear cell renal cell carcinoma. J Cell Biochem. 2018;119(12):10406-10414. doi:10.1002/jcb.27389

23. Zhou Y, Wang R, Xu T, et al. Prognostic value of long noncoding RNA CRNDE as a novel biomarker in solid cancers: an updated systematic review and meta-analysis. $J$ Cancer. 2019;10 (11):2386-2396. doi:10.7150/jca.31088

24. Ji D, Jiang C, Zhang L, et al. LncRNA CRNDE promotes hepatocellular carcinoma cell proliferation, invasion, and migration through regulating miR-203/BCAT1 axis. J Cell Physiol. 2019;234 (5):6548-6560. doi:10.1002/jcp. 27396

25. Tang D, Zhao L, Peng C, et al. LncRNA CRNDE promotes hepatocellular carcinoma progression by upregulating SIX1 through modulating miR-337-3p. J Cell Biochem. 2019;120(9):16128-16142. doi: $10.1002 /$ jcb. 28894
26. Wang G, Pan J, Zhang L, et al. Long non-coding RNA CRNDE sponges miR-384 to promote proliferation and metastasis of pancreatic cancer cells through upregulating IRS1. Cell Prolif. 2017;50(6). doi:10.1111/cpr.12389.

27. Huan J, Xing L, Lin Q, et al. Long noncoding RNA CRNDE activates $\mathrm{Wnt} / \beta$-catenin signaling pathway through acting as a molecular sponge of microRNA-136 in human breast cancer. Am J Transl Res. 2017;9(4):1977-1989.

28. Dai J, Mu J-W, Mu H. Long non-coding RNA CRNDE regulates cell proliferation, migration, invasion, epithelial-mesenchymal transition and apoptosis in oral squamous cell carcinoma. Oncol Lett. 2019;17 (3):3330-3340. doi:10.3892/ol.2019.9978

29. Song K, Liu N, Yang Y, et al. Regulation of osteosarcoma cell invasion through osteopontin modification by miR-4262. Tumour Biol. 2016;37(5):6493-6499. doi:10.1007/s13277-015-4530-8

30. Sun H, Zhou X, Bao Y, et al. Involvement of miR-4262 in paclitaxel resistance through the regulation of PTEN in non-small cell lung cancer. Open Biol. 2019;9(7):180227. doi:10.1098/rsob.180227

31. Zhang D, Li Z, Zhang Y, et al. miR-4262 promotes the proliferation of human cutaneous malignant melanoma cells through KLF6-mediated EGFR inactivation and p21 upregulation. Oncol Rep. 2016;36(6):3657-3663. doi:10.3892/or.2016.5190

32. Wang K, Ren Y, Liu Y, et al. miR-4262 promotes proliferation and invasion of human breast cancer cells through directly targeting KLF6 and KLF15. Oncol Res. 2017;25(2):277-283. doi:10.3727/ 096504016X14732514133203

33. Zhang H, Jiang H, Zhang H, et al. miR-4262, low level of which predicts poor prognosis, targets proto-oncogene CD163 to suppress cell proliferation and invasion in gastric cancer. Onco Targets Ther. 2019;12:599-607. doi:10.2147/OTT.S187881

34. Qu JJ, Qu XY, Zhou DZ. miR4262 inhibits colon cancer cell proliferation via targeting of GALNT4. Mol Med Rep. 2017;16 (4):3731-3736. doi:10.3892/mmr.2017.7057

\section{Publish your work in this journal}

OncoTargets and Therapy is an international, peer-reviewed, open access journal focusing on the pathological basis of all cancers, potential targets for therapy and treatment protocols employed to improve the management of cancer patients. The journal also focuses on the impact of management programs and new therapeutic agents and protocols on patient perspectives such as quality of life, adherence and satisfaction. The manuscript management system is completely online and includes a very quick and fair peer-review system, which is all easy to use. Visit http://www.dovepress.com/ testimonials.php to read real quotes from published authors. 